I. Göcze

W. A. Wohlgemuth

H. J. Schlitt

E. M. Jung

\section{Contrast-enhanced ultrasonography for bedside imaging in subclinical acute kidney injury}

Received: 24 October 2013

Accepted: 30 October 2013

Published online: 16 November 2013

(C) Springer-Verlag Berlin Heidelberg and ESICM 2013

I. Göcze (®) · W. A. Wohlgemuth · H. J. Schlitt · E. M. Jung Departments of Surgery and Radiology, University Medical Center Regensburg, Franz-Josef-Strauss-Allee 11, 93042 Regensburg, Germany

e-mail: ivan.goecze@ukr.de

A 26-year-old woman with complete intra- and extrahepatic thrombosis of the portal vein underwent surgical thrombectomy. The patient received a portocaval shunt and consecutively an angiographic catheter into the portal vein for catheter-directed continuous thrombolysis. Twelve hours after admission to ICU, an angiography examination

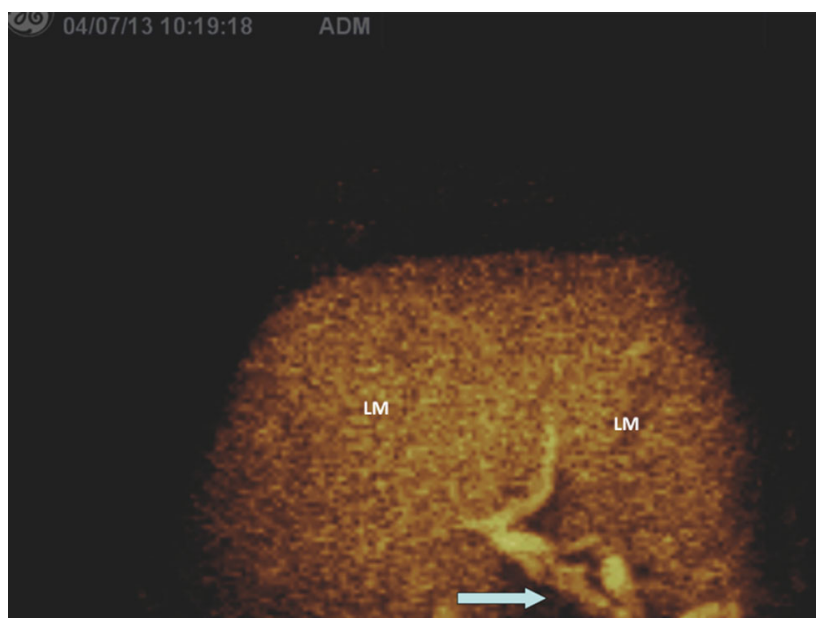

Fig. 1 Contrast-enhanced ultrasonography (CEUS) imaging of the liver showing reduced and inhomogeneous enhancement of the portal vein as a typical finding for partial thrombosis (arrow). $L M$ liver microvascularisation

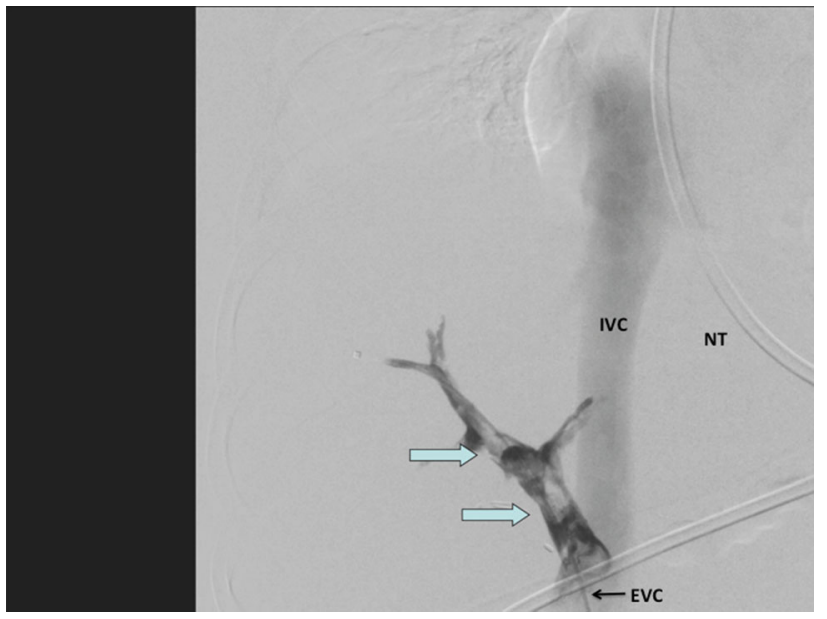

Fig. 2 Angiography via endovascular catheter $(E V C)$ with filling defects (white non-contrasted areas) in the portal vein with partial thrombosis (arrows), correlating with CEUS findings. IVC inferior cava vein, NT nasogastric tube

was scheduled for re-assessing liver circulation. At this time, she was mechanically ventilated and required inotropic support. Because of her serious condition with subclinical acute kidney injury (AKI), the scheduled angiography was postponed and the application of a nephrotoxic contrast agent avoided. Instead, the patient was examined by means of contrast-enhanced ultrasonography (CEUS) at the bedside. The microbubble contrast agent without any nephrotoxic effect was injected via endovascular catheter and also intravenously. The bedside images showed improved perfusion in the main portal vein with only partial thrombosis (Fig. 1). The CEUS results corresponded well with the findings of angiography examination conducted the next day (Fig. 2).

Written consent for publication was obtained from the patient.

Conflicts of interest The authors declare that they have no conflicts of interest. 